\title{
Collective single pass gain in a tunable rectangular grating amplifier
}

\author{
L. J. Louis, J. E. Scharer, and J. H. Booske \\ Electrical and Computer Engineering Department, University of Wisconsin, Madison, Wisconsin 53706
}

(Received 30 September 1997; accepted 7 April 1998)

\begin{abstract}
The shape of the gain curve and physics of interaction between microwave fields and the electron beam for a periodic rectangular grating slow-wave Traveling Wave Tube (TWT) amplifier are investigated. This research focuses on the collective $\left(\omega_{p} \tau \gg 1\right)$ low-voltage $(\leqslant 10 \mathrm{kV})$ interaction in the backward wave regime for the Ku-band $(12.5-17.5 \mathrm{GHz})$ range of frequencies. For amplifier experiments, a round "probe" beam $(10 \mathrm{kV}, 0.25 \mathrm{~A}, 1 \mathrm{~mm}$ diameter $)$ confined by a $1 \mathrm{kG}$ solenoidal focusing magnetic field was utilized. Simultaneous single-pass collective gain and electron-beam velocity spread measurements are performed by means of a Faraday cup repeller energy analyzer configured as the beam collector. Experiments near $13.0 \mathrm{GHz}$ show the maximum normalized single-pass gain to be in agreement with the theoretical prediction for several axial velocity spreads $\left(\delta v_{\|} / v_{\|}\right)$. Collective single-pass experimental results are presented and compared with theory. The effect of an increased interaction length which is accomplished by partial end reflections on the enhanced convective growth is measured and discussed. Narrowing of the gain curve as a function of beam voltage and frequency is shown to result from critical details of the electron-beam radial current density distribution and is verified experimentally by varying the beam-grating separation. Experimental results for several beam-grating separations and the effect of a nonuniform peaked radial current density on the interaction physics are also discussed. (C) 1998 American Institute of Physics. [S1070-664X(98)02807-9]
\end{abstract}

\section{INTRODUCTION}

The rectangular grating amplifier is being investigated for use as a compact, low-voltage, moderate power millimeter wave coherent radiation source. This paper presents an experimental investigation of the shape of the beam-energydependent, collective, single-pass gain for the backward wave tunable grating amplifier. The rectangular grating utilized in the previous experiments ${ }^{1-3}$ employed electron beam energies $\geqslant 100 \mathrm{kV}$. These configurations were either open grating structures without sidewalls or an axial cavity resonator and the grating modes can be expanded in terms of transverse electric (TE) and transverse magnetic (TM) modes.

In our previous paper ${ }^{4}$ on the theoretical analysis of wave dispersion and growth, the modes for the present closed rectangular waveguide with a grating on one of its broad walls (Fig. 1) are expanded in terms of hybrid modes ${ }^{5}$ transverse electric to $x\left(\mathrm{TE}_{x m n}\right){ }^{6}$, where $x$ refers to the transverse dimension of the guide. The other set of hybrid modes, $\mathrm{TM}_{x}$, operates at much higher frequencies. A sheet beam configuration $^{7}$ is compatible with the rectangular grating geometry and would enhance the effective interaction between grating modes and electron beam. To study the physics of the interaction, a low energy $(\mathrm{V} \leqslant 10 \mathrm{kV})$ round "probe" beam is utilized for experimental observations. Phase measurements and preliminary gain measurements previously reported $^{8}$ verified some of the important features of the theoretically predicted electromagnetic wave dispersion and growth.

The collective single-pass gain discussed in this paper was measured with an improved method for input micro- wave coupling. An E-Plane Tee was used for input coupling, providing a stronger microwave pump. The ability to vary the electron beam energy continuously provided a means to study the shape of the experimental gain curve as a function of beam-energy. The use of an annular Faraday cup energy analyzer along with the collector configuration enables simultaneous gain and beam spread measurements. The dependence of gain on beam axial velocity spread is discussed and compared to theoretical predictions.

Collective space-charge effects ${ }^{9}$ dominate the interaction for this device, since the transit time is longer than a plasma period. Using asymptotic (Fourier) analysis we calculate the growth rate for $\mathrm{TE}_{x}$ grating modes interacting with an electron beam with Maxwellian axial velocity spread. The experimentally measured peak value of single-pass gain is found to match the theoretically predicted value. The line shape of the experimental gain differs from theory in two respects listed below:

(1) The experimental gain curve as a function of beam voltage is narrower than theory. This corresponds to a narrowing in the bandwidth of operation.

(2) Theoretically predicted enhanced convective growth at higher beam energies is reduced from the predicted value in the single pass gain measurements.

Detailed experimental studies were conducted to explain these differences. To study the narrowing of the gain curve the effect of electron-beam radial current density distribution on the gain function was analyzed. The theory considers the electron-beam radial current density to be uniform, while knife edge experiments have shown the radial current density profile to be Gaussian. Beamlets and different beam profiles 


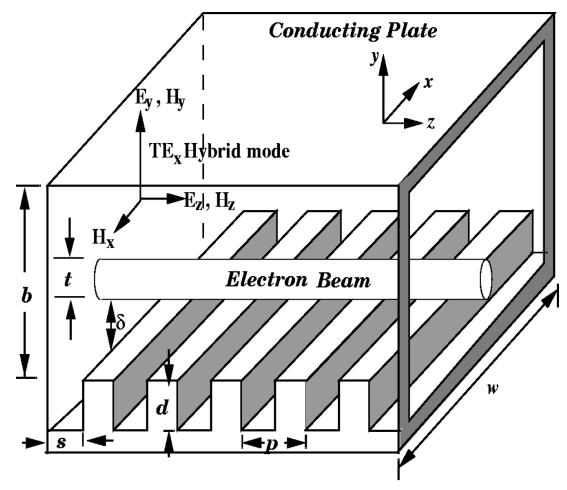

FIG. 1. Rectangular waveguide grating schematic.

are used to study the effect of the peaked (Gaussian) current density distribution on the gain function. By varying the beam-grating separation the interaction physics between an exponentially decaying grating mode and an electron beam with Gaussian radial density profile is studied. Experimental results for several beam-grating separations are presented.

In the analysis of the backward wave regime, where the beam and wave phase velocities are in opposite directions, the monoenergetic beam theory predicts a dominant absolute instability $^{10}$ at a beam energy larger than the beam-wave velocity-synchronism axial energy, as well as a convective growth at beam energies closer to the beam-wave velocitysynchronism. As the fractional axial velocity spread is increased above 0.01, the absolute instability is transformed into convective growth and manifests as "enhanced convective growth." This enhanced convective growth is theoretically observed as a knee in the theoretical gain-versus-beamenergy function. However, its presence in the experimentally measured gain function is significantly less than the theoretical prediction. In an effort to observe the enhanced convective growth, the effective interaction length for the present system is increased by adding partial end reflections. Partial reflections produce a low-Q multiple-pass interaction for the microwaves while allowing the beam to pass through the system. Experimental results for the increased-interactionlength (multiple-pass) system are discussed.

In Sec. II, the experimental single-pass gain as a function of beam spread is compared with theoretical predictions. Also the effect of increased interaction length in the multiple-pass system on the gain shape is discussed and compared with the experimental single-pass and theoretical gain. In Sec. III, the sensitivity of the single-pass gain shape for different beam/grating separations and the radial profile of the beam current density distribution is discussed. A summary section concludes the paper.

\section{SINGLE-PASS GAIN MEASUREMENT}

The grating amplifier consists of a 10.5 in., 75 period grating segment attached to the bottom of a channel block, producing a grating-lined $\mathrm{Ku}$-band rectangular-cross-section waveguide as shown in Fig. 1. The grating has a period $p=3.556 \mathrm{~mm}$, groove width $s=1.778 \mathrm{~mm}$ and groove depth $d=1.588 \mathrm{~mm}$. The electron beam has a diameter of $t \simeq 1$

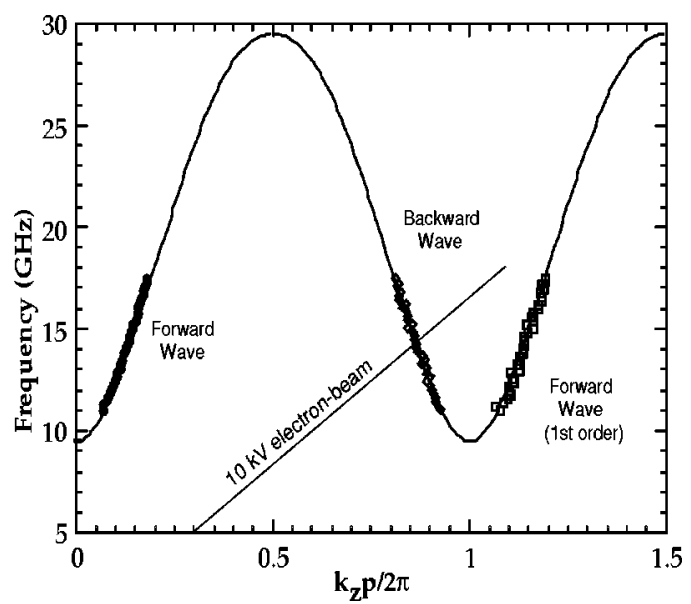

FIG. 2. Dispersion diagram for the $\mathrm{TE}_{x 10}$ hybrid mode.

$\mathrm{mm}$. The uncoupled (beam-free) dispersion diagram ${ }^{8}$ for the rectangular grating waveguide is shown in Fig. 2. The dots correspond to experimental measurements of both the fundamental spatial-harmonic and the first-order spatial-harmonic for the fundamental hybrid mode $\left(\mathrm{TE}_{x 10}\right)$ in the Ku-band frequency range. The single-pass gain measurements describe the interaction between a low energy $(\mathrm{V} \leqslant 10 \mathrm{kV})$ electron-beam and the backward wave fundamental spatial harmonic of this $\mathrm{TE}_{x 10}$ hybrid mode. The collective growth or absorption is measured as a function of beam-energy at a fixed frequency.

\section{A. Experimental setup}

The grating amplifier system is placed inside a vacuum chamber whose base pressure is $10^{-8}$ Torr and is supported by two linear motion transducers so that an accurate vertical alignment can be made. Two additional linear motion transducers are attached to the grating utilizing U-shape clamps to allow a horizontal position adjustment. These vacuum feedthroughs allow the grating position to be adjusted with respect to the electron beam during the course of experiments. Figure 3 illustrates the side view of the grating amplifier system in the vacuum chamber with the magnetic focusing system.

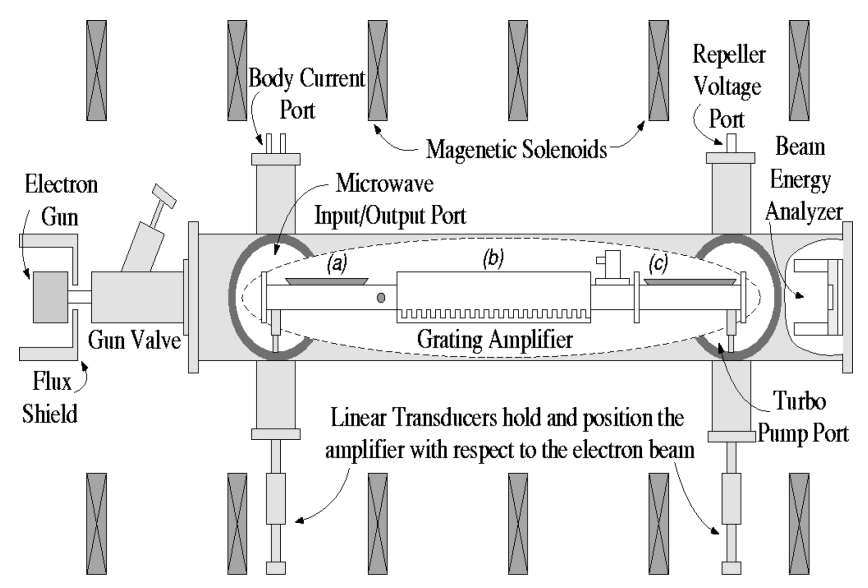

FIG. 3. Side view of grating amplifier. 


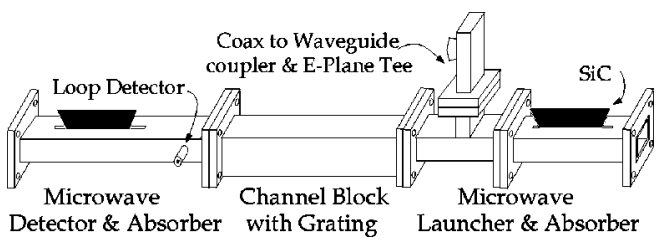

FIG. 4. Single-pass backward wave grating amplifier setup.

The single-pass grating amplifier has three sections and its schematic is shown in Fig. 4. These three sections of the grating are electrically isolated from each other to measure the individual body currents produced by the beam interception. Microwave input power is coupled to the system by means of an E-Plane Tee connected to the upstream-end of the grating-lined waveguide. The output section consists of a smooth-wall Ku-band waveguide with a magnetic loop detector on the narrow wall of the waveguide and is attached to the downstream-end of the grating-lined waveguide to detect the amplified signal. A smooth-wall Ku-band waveguide with a tapered silicon carbide absorber inserted in the centered broadwall slots is connected at either end of the system to suppress reflections.

To allow for the desired movement of the grating system with respect to the electron beam inside the compact vacuum chamber, the length of the E-plane-arm of the tee was reduced. However, network analyzer measurements showed only a small change in the transmission and reflection properties of the tee due to this reduction of the E-plane-arm height. For example, the transmission parameter $S_{21}$, with port 1 designating the E-plane-arm, at $13.0 \mathrm{GHz}$ was found to be $-3.68 \mathrm{~dB}$ compared to an idealized parameter of -3.0 $\mathrm{dB}$ for a conventional (longer-arm) tee. Figure 5 shows the external circuitry used for microwave launching and detection for the waveguide-coupled single-pass case.

\section{B. Beam axial velocity spread measurements}

The modified collector configuration shown in Fig. 3 includes a Faraday cup energy analyzer to enable simultaneous gain and velocity spread measurements. The collector is coated with graphite to suppress secondary emission. ${ }^{11} \mathrm{~A}$ typical oscilloscope trace of the beam spread measurement is shown in Fig. 6. The repeller voltage is varied about the beam voltage to produce a variation in the collected current from zero to its maximum. A $4 \mathrm{kV}$ oscillating voltage is

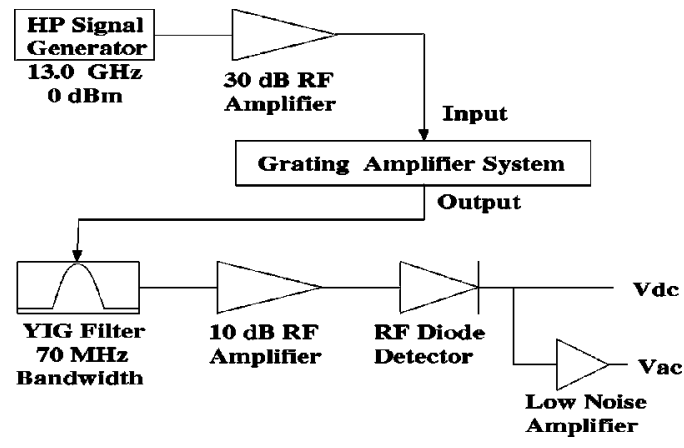

FIG. 5. External rf circuitry for single-pass system.
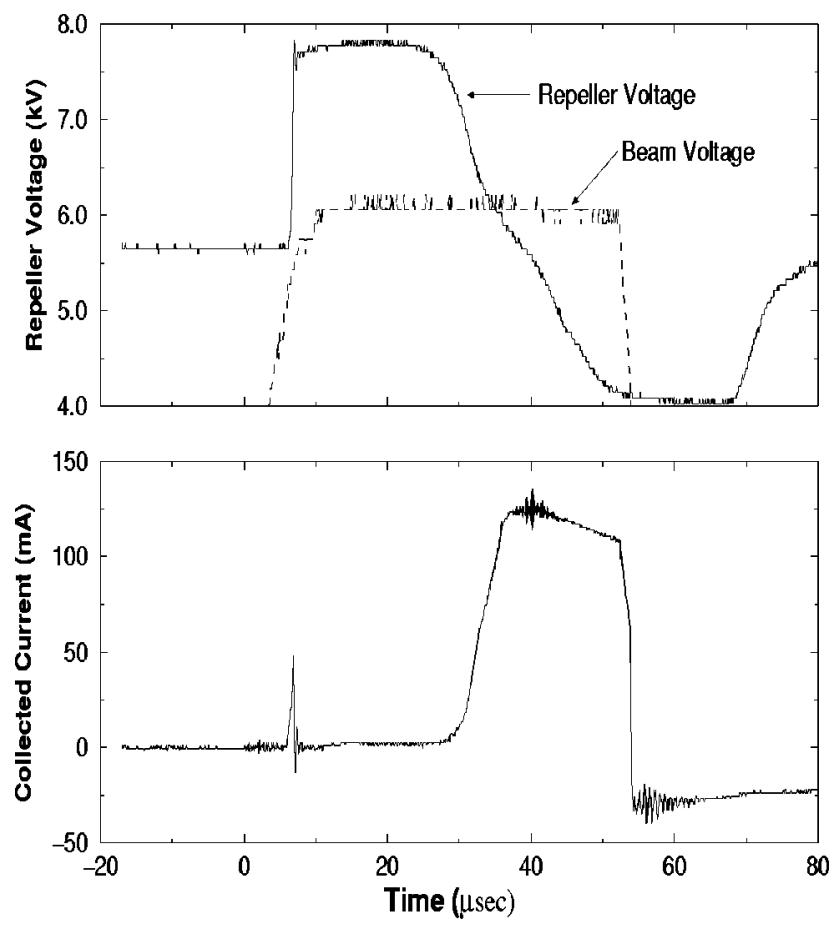

FIG. 6. Typical oscilloscope trace of repeller voltage and collector current.

superimposed on the dc repeller voltage. The plot of the collector current $\left(I_{C}\right)$ as a function of the repeller voltage $\left(V_{R}\right)$ is shown in Fig. 7. The derivative of the collected current with respect to the repeller voltage (i.e., $d I_{C} / d V_{R}$ ) is superimposed on the plot. This gives the beam axial velocity distribution function $f\left(V_{b}\right)$. By using the expression $\delta v_{\|} / v_{\|}=\delta V_{b} / 2 V_{b}$, the fractional electron beam axial velocity spread for this case is determined to be 0.036 .

\section{Experimental single-pass gain results}

Figure 8 shows a typical oscilloscope trace for the gain measurements. The top portion of the figure shows the varia-

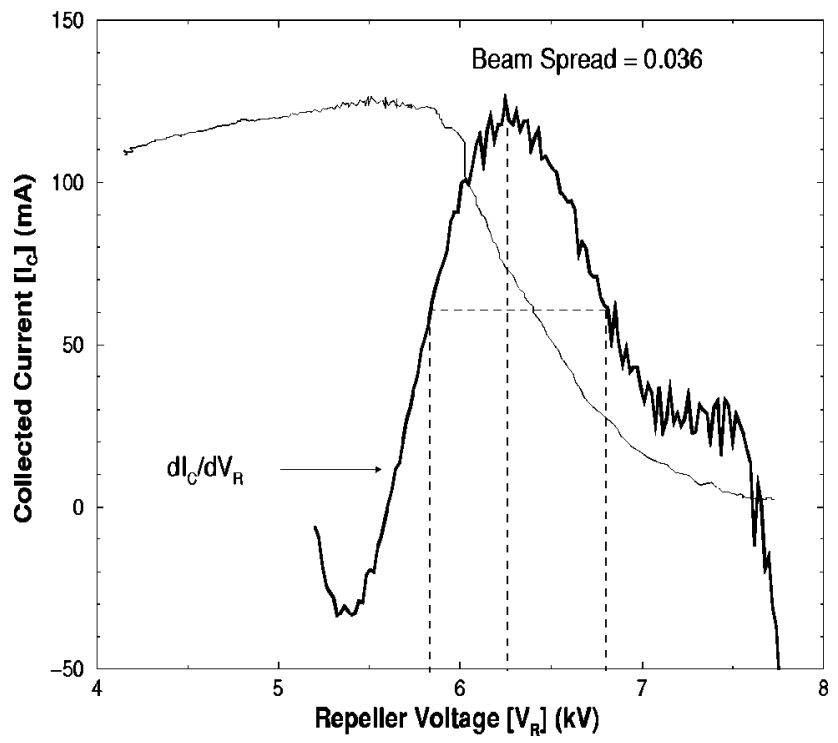

FIG. 7. Plot of collector current vs repeller voltage. 

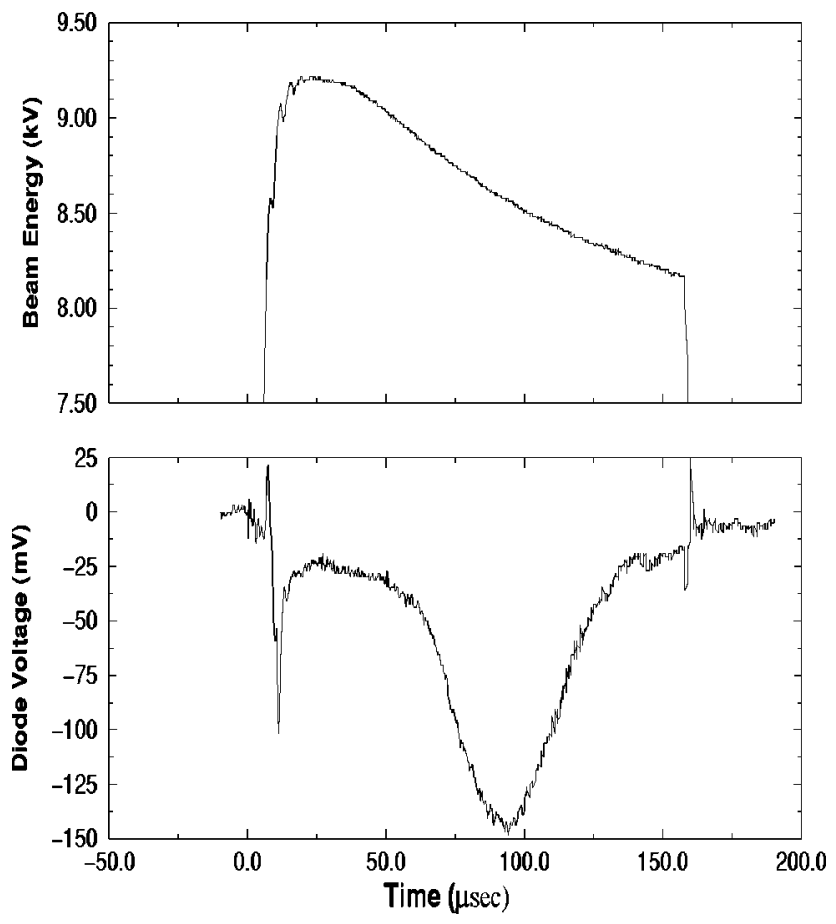

FIG. 8. Oscilloscope trace of typical gain curve.

tion in the electron beam energy as a function of time and the bottom portion shows the corresponding change in the output voltage of the crystal detector after being amplified by the low noise amplifier. This is the change in gain produced by the change in electron beam energy. The spikes in the diode voltage correspond to the transient rise and fall portions of the beam energy. This spike is a sinusoidal swing denoting absorption and gain similar to the gain curve and is produced by the beam energy moving from zero to its maximum value during the rise and vice-versa during the fall.

The normalized $\left(\left(P_{\text {out }}-P_{\text {in }}\right) / P_{\text {in }}\right)$ gain for the singlepass operation is shown in Fig. 9 for two beam spreads at a fixed frequency of $13.0 \mathrm{GHz}$. The dilute-beam-wave velocity

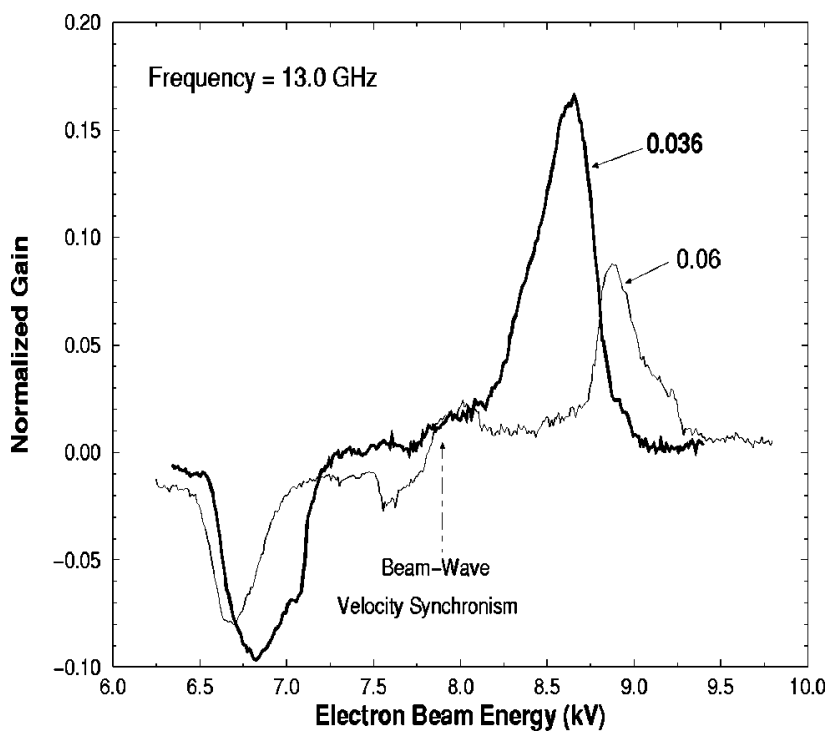

FIG. 9. Experimental single-pass gain for two beam spreads.

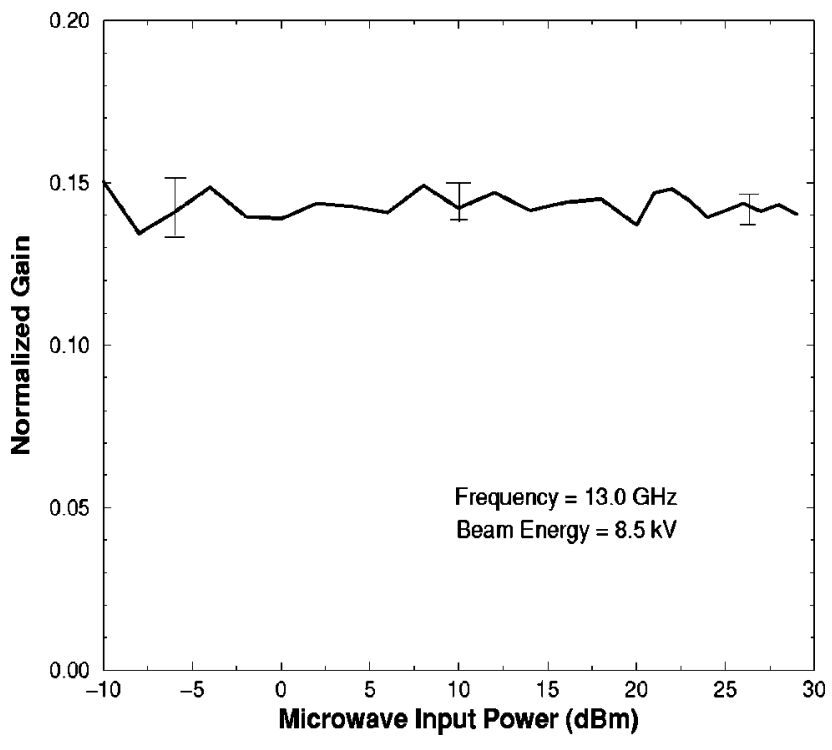

FIG. 10. Experimental gain for varying input power levels.

synchronism condition given by $\omega \simeq k_{z} v_{0}$ occurs at $7.85 \mathrm{kV}$. For a finite-thickness beam in a bounded waveguide, there are an infinite number of propagating space-charge normal-modes ${ }^{12,13}$ on the beam. The beam space-charge waves with frequencies $k_{z} v_{0}-\omega_{p e}<\omega<k_{z} v_{0}$ are the "slow space charge waves" (SSCW), while those space-charge waves in the range $k_{z} v_{0}<\omega<k_{z} v_{0}+\omega_{p e}$ are the "fast space charge waves" (FSCW). There is gain for beam energies greater than that for beam resonance which interact with the SSCW and absorption for beam energies less than beam resonance which interact with the FSCW.

The plasma frequency $\left(f_{p}\right)$ for this device is $1.54 \mathrm{GHz}$, giving a plasma period $\left(t_{p}=1 / f_{p}\right)$ of $0.64 \mathrm{~ns}$. For the interaction length $(L)$ of $27 \mathrm{~cm}$, the transit time $\left(\tau=L / v_{o z}\right)$ is 5 ns. Since the transit time is significantly longer than a plasma period, the collective space-charge-wave effects are important to the interaction. ${ }^{9}$ The corresponding normalized space charge parameter $\left(\theta_{p}=\omega_{p} \tau\right)^{14}$ for this device is found to be 48.

It is observed in Fig. 9 that, as expected, the experimentally measured maximum gain or absorption decreases as the velocity spread increases. The beam energy experiencing maximum gain or absorption can be seen to shift further away from beam-wave velocity synchronism as the velocity spread increases.

\section{Linearity in gain measurements}

To study the linearity of the gain with input power levels, measurements are carried out at a fixed electron beam energy and fixed frequency for a wide range of input power levels. Figure 10 shows the variation of normalized gain for wide range of input power levels. The frequency is fixed at 13.0 $\mathrm{GHz}$ and the beam energy producing the peak gain is fixed at $8.6 \mathrm{kV}$. The beam has an axial velocity spread of 0.04. The normalized gain is constant within the limits of 


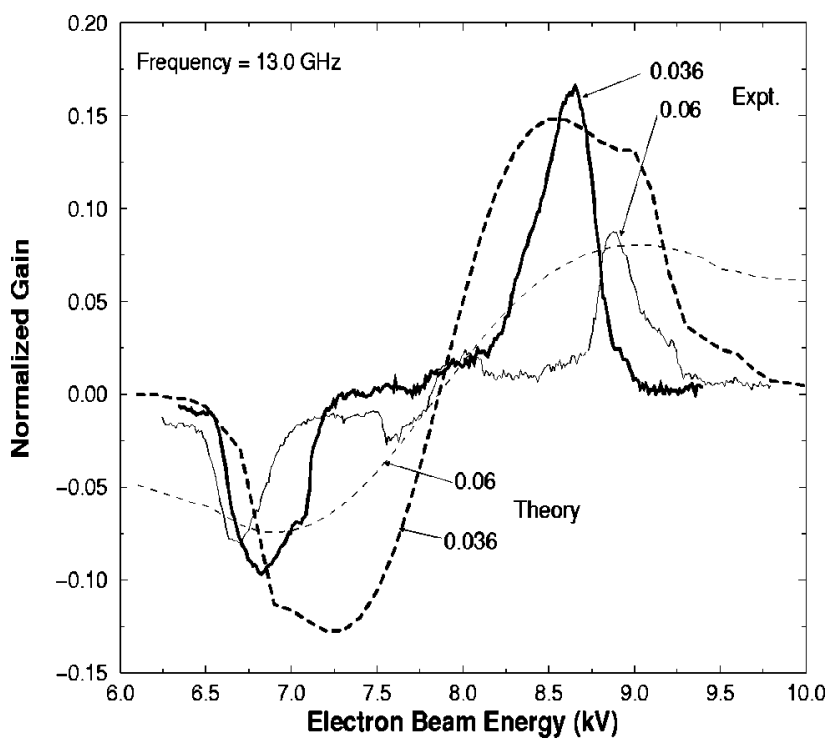

FIG. 11. Theoretical comparison of single-pass gain for corresponding beam spreads.

error for input microwave power levels from +30 to -10 $\mathrm{dBm}$. This in turn illustrates that the grating amplifier is operating in the linear regime. ${ }^{14}$

\section{E. Comparison with theoretical predictions}

The GRAting AMPlifier (GRAMP) ${ }^{4,6}$ code is used to obtain the theoretical growth rate per period for backward wave operation. This code is capable of solving the complex dispersion relation $[D(\omega, k)]$ for the complex $k\left(k_{r}+i k_{i}\right)$, where $k_{r}$ gives the dispersion diagram and $k_{i}$ gives the linear growth rate for a sheet beam interaction. Since collective space-charge-wave effects ${ }^{9}$ dominate the interaction for this device, asymptotic (Fourier) analysis is used in the GRAMP code to calculate the growth rate per period. To compare this with the experimental results obtained with a pencil beam, the theoretical growth rate per period is multiplied by the number of periods and a geometric filling factor. ${ }^{15}$

Figure 11 shows the comparison of experimental gain with theoretical predictions for corresponding beam spreads. The maximum value of the experimental single-pass gain corresponds closely to the theoretical value for both velocity spread cases. The theoretical result predicts that the amount of absorption is lower than the amount of gain, since the interacting beam-current density $\left(J_{b}\right)$ depends on the beam voltage $\left(V_{b}\right)$. This dependence is given by $J_{b} *$ Area $=P / V_{b}^{3 / 2}$, where $P=0.25 \mu$ pervs is the beam perveance for the experimental electron beam.

There are differences in the shape of the experimental gain curve when compared with the theory. It is noted that the shape of the experimental gain curve is narrower than the theoretical prediction and the reasons for this are discussed in the next section. The gain portion of the experimental curve lies within the theory curve, however, its peak matches the theoretical prediction. The absorption portion of the experimental curve, however, differs from theoretical predictions as follows. The maximum measured absorption is re-

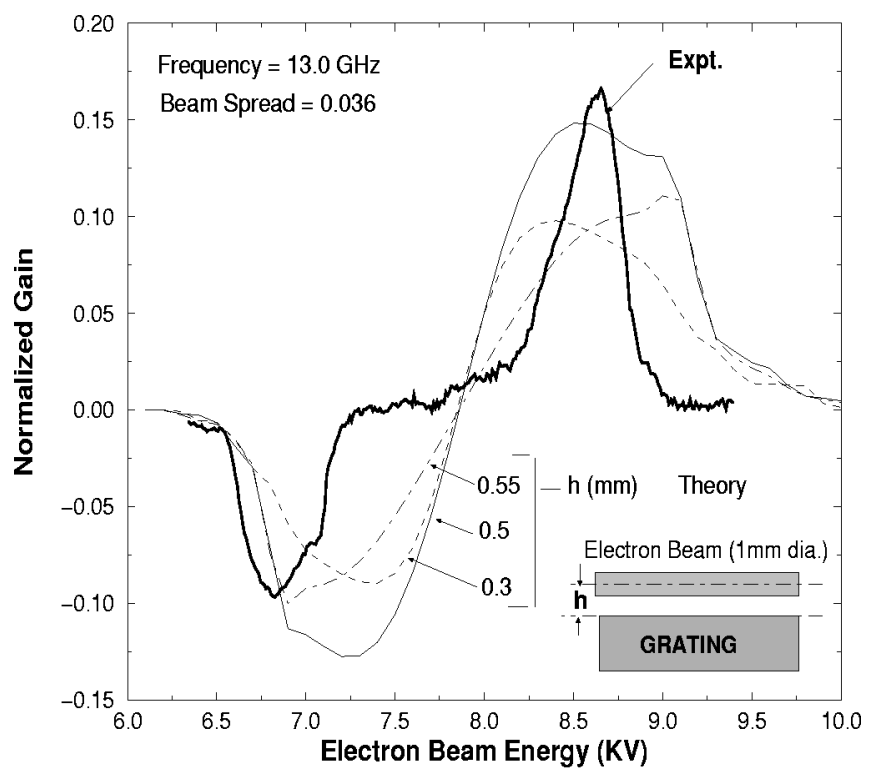

FIG. 12. Theoretical comparison of single-pass gain for different beam separations.

duced and the peak absorption occurs at a beam energy lower than theoretical predictions. The reason for this result is that the beam-grating separation is subject to a small variation as the beam energy is swept through the growth and absorption regimes. Due to beam entrance optics effects, the beamgrating separation increases approximately by $0.05 \mathrm{~mm}$ as the beam energy is lowered from 8.5 to $7 \mathrm{keV}$. This energydependent increase in the beam-grating separation is also observed in the experimental body current measurements as a reduction in the grating-intercepted body current, for lower beam energies. Even though this variation is small in magnitude, a slight increase in the beam-grating gap has a substantial effect on the shape of absorption and growth curves as is evident from the following theoretical comparison.

Figure 12 compares the experimental gain for a fractional velocity spread of 0.036 with theoretical predictions for several beam-grating separations. Here $h$ is the distance of separation of the electron-beam axis to the grating surface and the experimental beam has a radius $\left(r_{b}\right)$ of $0.5 \mathrm{~mm}$. It shows that the peaks of the gain and absorption portion shift further away from the beam resonance point as the beamgrating separation increases $\left(h>r_{b}\right)$ and shift towards the beam resonance point as the beam-grating separation decreases $\left(h<r_{b}\right)$. This illustrates that the experimentally measured absorption peak shifted to a lower beam energy because the beam-grating separation for the absorption portion was slightly greater than that for the gain portion. The hybrid $\mathrm{TE}_{x m n}$ modes $(m=1,2, \ldots, n=0,1,2, \ldots)$ inside the structure are surface harmonic modes where the electromagnetic fields are guided by the grating surface and the field strength decays exponentially away from grating surface. ${ }^{4}$ Hence, larger beam-grating spacing experiences reduced interaction. On the other hand a reduction in the amount of gain or absorption as the beam-grating spacing decreases occurs due to a reduction in the total interacting beam current since the beam is shaved by the grating surface. 


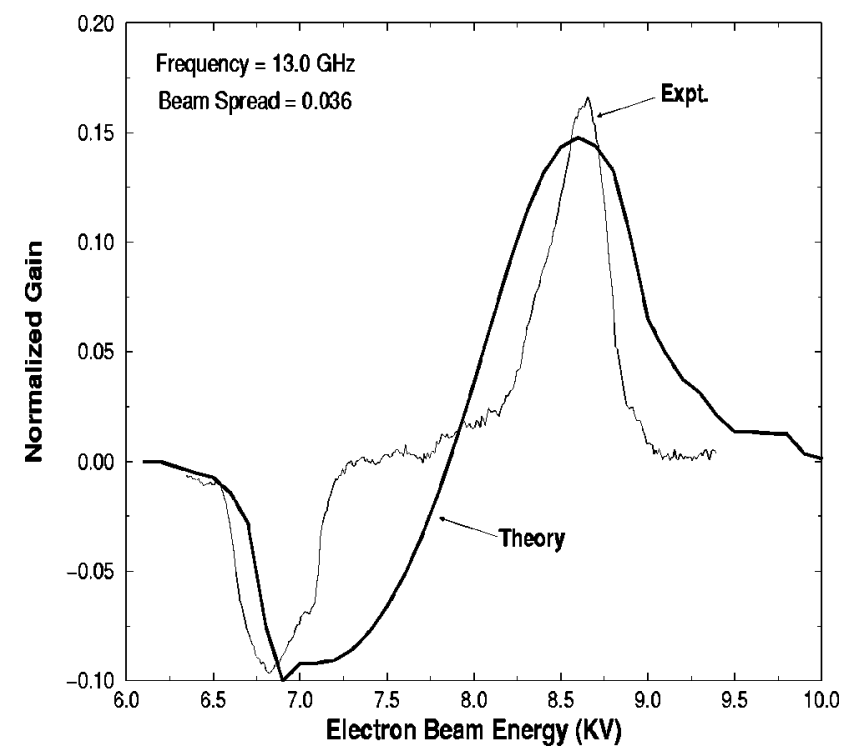

FIG. 13. Theoretical comparison of single-pass gain with modified theory.

To theoretically verify these explanations for the asymmetrical details in the gain-versus-beam-energy function about the beam-wave velocity synchronism point, the theory, which previously assumed uniform beam-grating separation at all beam energies was modified to account for this variation $\left[h=\right.$ function $\left.\left(V_{b}\right)\right]$. The modified theory considers a linear variation of beam-grating separation $(h)$, with $h=0.55$ $\mathrm{mm}$ at the absorption peak $\left(V_{b}=6.8 \mathrm{kV}\right)$ and $h=0.5 \mathrm{~mm}$ at the gain peak $\left(V_{b}=8.6 \mathrm{kV}\right)$. Figure 13 compares the experimental gain with the modified theory. The entire theoretical curve, including the absorption portion, shows much better agreement with the experimental curve than Fig. 11.

\section{F. Increased interaction length by partial end reflections}

The shape of the theoretical gain curve in Fig. 11 for the 0.036 beam spread case has a knee due to enhanced convective growth at a beam energy of $9.2 \mathrm{kV}$ in addition to the peak convective growth at $8.6 \mathrm{kV}$. As the axial velocity spread increases the dominant absolute instability for the backward wave regime is transformed into enhanced convective growth and its magnitude decreases with increasing axial velocity spread, due to Landau damping. This is observed as a broadening of the theoretical gain shape as a function of beam energy. However, this enhanced convective growth is an asymptotic effect, and its presence may not be as pronounced for single-pass measurements, depending on the length of the grating-beam interaction. Hence increasing the effective interaction length in the experimental system should increase the magnitude of the enhanced convective growth. In an effort to observe this enhanced growth, partial end reflections were introduced to produce an effective increase in the interaction length. Using partial reflections results in a low-Q multiple-pass system which cannot produce spontaneous oscillation, but is similar to increasing the length of the interaction.

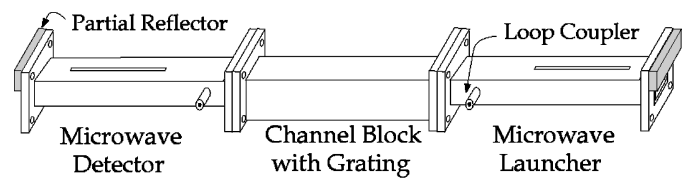

FIG. 14. Multiple-pass backward wave grating amplifier setup.

The schematic of the experimental multiple-pass system is shown in Fig. 14. The silicon carbide absorbers are removed and partial reflectors are added to both ends. Partial reflection is produced by covering the upper half of the waveguide with a copper sheet, leaving the grating lined bottom half of the waveguide open for electron beam interaction. The waveguide tee input coupler is replaced by a loop coupler, to reduce the microwave power leaving the cavity through the tee and to avoid complex cavity effects. Hence this multiple-pass has a lower input microwave coupling than the previously discussed single-pass system.

The normalized experimental gain for multiple-pass operation is shown in Fig. 15 for two beam spreads. A maximum multiple-pass gain of 0.22 is achieved for a velocity spread of 0.036 compared to 0.16 in the single-pass case. This shows the presence of modestly enhanced wave-beam interactions in the multiple-pass system.

Figure 16 shows the comparison between single-pass and multiple-pass experimental gain curves with asymptotic theoretical predictions for a 0.036 velocity spread. To illustrate the difference in the line shape of the gain curves the peaks are re-normalized. The multiple-pass and single-pass experimental curves are similar except for beam energies in the vicinity of the theoretically predicted enhanced convective growth knee. In the 8.7 to $9.4 \mathrm{kV}$ beam energy range the shape of the multiple-pass gain curve is significantly enhanced in comparison to the single-pass curve. This shows the added sensitivity of the increased interaction length in the multiple-pass system to the theoretically predicted enhanced

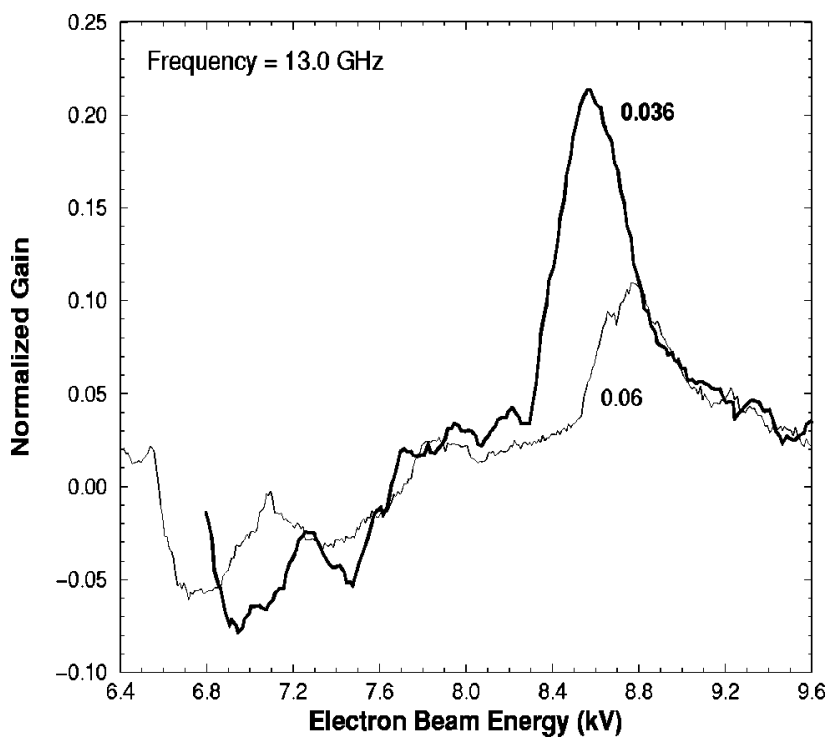

FIG. 15. Experimental multiple-pass gain for two beam spreads. 


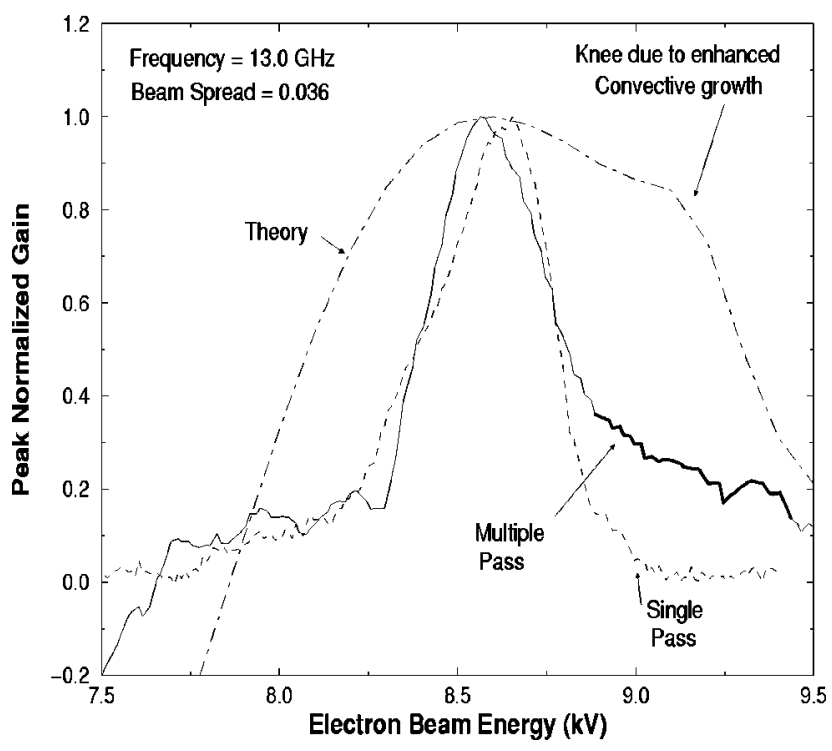

FIG. 16. Comparison of peak normalized single and multiple pass gain.

convective growth, even though the experimental manifestation is still smaller than the theoretical prediction.

\section{GAIN FOR SEVERAL BEAM-GRATING SEPARATIONS}

The shape and position of the theoretical gain peak can be seen to vary for different beam-grating separations as shown in Fig. 12. This would manifest as similar functional effects in frequency space for an amplifier subjected to variable input frequency with a fixed beam energy. Thus the bandwidth and gain can be varied by varying the beamgrating separation. To experimentally verify the shift in the shape and position of gain peaks, and to explain the narrowing of the experimental gain curves, single-pass gain measurements were done for several beam-grating separations.

\section{A. Effects of electron-beam current density distribution}

The electron-beam current density radial profile is assumed to be uniform in the theory ${ }^{4}$ for calculating dispersion and growth. But the experimental beam is measured to have an approximate Gaussian distribution for the radial current density profile. To incorporate the effect of a nonuniform current density profile, the beam is envisioned to be made of several thin beamlets and the growth for these beamlets are evaluated separately. Figure 17 shows the gain for a beam (with diameter $1 \mathrm{~mm}$ ) split into four beamlets. Let the current density of the outer (\#1 and \#4) beamlets be $J_{\text {ext }}$ and that of inner (\#2 and \#3) beamlets be $J_{\text {int }}$. To approximately model a peaked (Gaussian) distribution, $J_{\text {ext }}$ is taken to be less than $J_{\text {int }}$. The peak-gain contribution from a beamlet (\#1) closer to the grating is closer to beam-wave velocity synchronism, and the contribution from a beamlet (\#4) away from the grating is farther away from the beam-wave velocity synchronism. So as the radial current density profile for a nonuniform beam becomes narrower, the magnitude of $J_{\text {int }} / J_{\text {ext }}$ increases. This results in a reduction in the gain

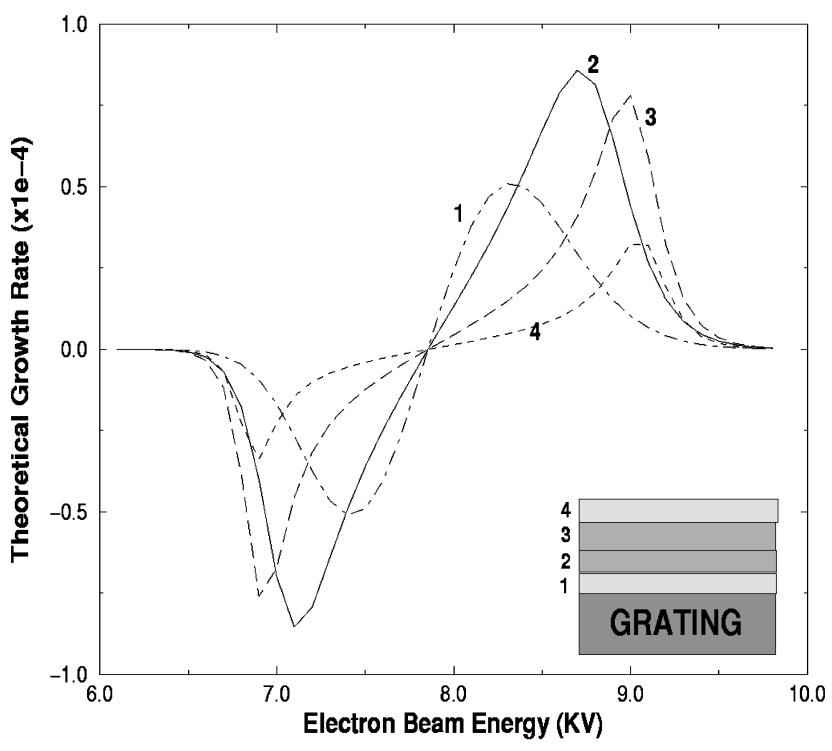

FIG. 17. Individual theoretical gain for the beamlets of a non-uniform beam.

contribution by the outer beamlets and an increase in the gain contribution from the inner beamlets. This produces a narrowing of the gain-versus-energy function from that predicted by a uniform beam model that uses the actual rms beam radius for the model radius of the uniform beam theory.

To model the effect of a more peaked current density distribution with the GRAMP code, the gain function of two profiles are compared. Profile 1 is a beam with a model diameter of $1 \mathrm{~mm}$ and a uniform current density $\left(J_{1}\right)$ of 25 $\mathrm{A} / \mathrm{cm}^{2}$ at $10 \mathrm{kV}$ beam energy. Profile 2 is a beam whose model diameter is reduced by $75 \%$, and to keep the total current in both beam profiles to be same, the value of $J_{2}$ is $1.33 J_{1}$. Both beams are positioned over the grating with beam-grating separation $(h)$ of $0.5 \mathrm{~mm}$. The theoretical gain for these two profiles are compared with the experimental single-pass gain in Fig. 18. It is noticed that the current density profile 2 with a narrower, more peaked distribution produces a narrower gain function than the current density profile 1 , and is therefore tending to closer agreement with the experimental shape. A dramatically narrower theoretical gain function could be obtained with even narrower beamlets (see Fig. 17).

\section{B. Experimental gain results for several beam-grating separations}

By slightly varying the beam-grating separation experimentally, the central portion of the electron beam which dominates the gain characteristics due to its increased current density could be moved closer to the grating's surface waves. Single pass gain was measured at a fixed frequency of $13 \mathrm{GHz}$ for the following four beam-grating spacings. The beam-grating separation $(h)$ in case-A is equal to the beam radius. Case-B is obtained by moving the beam closer to the grating with $h$ less than the beam radius $(h=0.4 \mathrm{~mm})$, and case-C moves the beam even closer to the grating $(h=0.3$ 


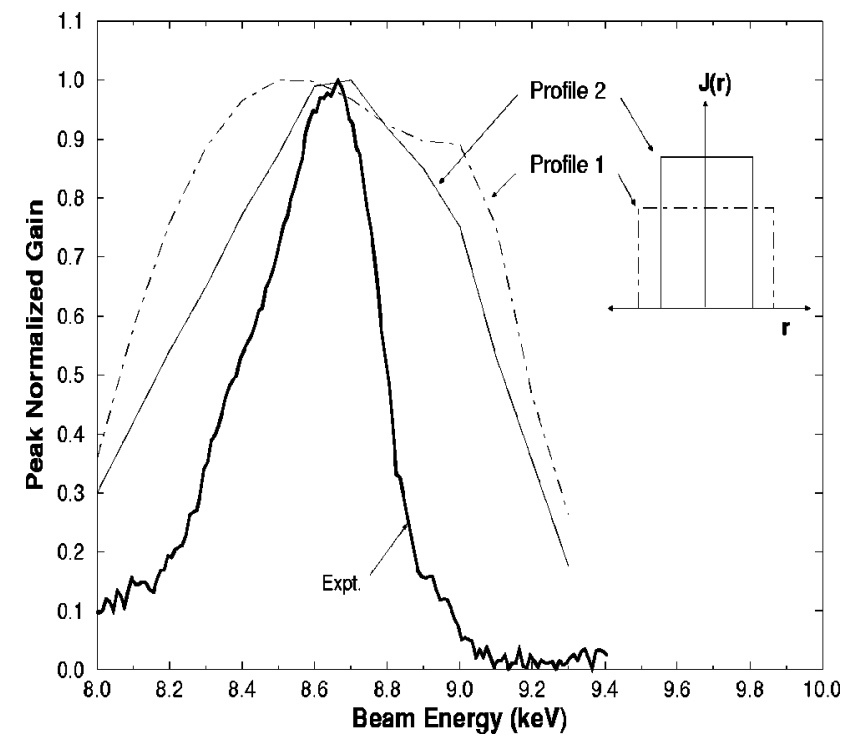

FIG. 18. Comparison of peak normalized single-pass gain for two radial current density profiles.

$\mathrm{mm})$. Case-D is obtained by moving the beam away from grating where $h$ is greater than the beam radius $(h=0.6 \mathrm{~mm})$.

The experimental gain curves for these four different cases are shown in Fig. 19. These results are for a fractional axial velocity spread of 0.036 . As expected, positioning the central portion of the electron beam closer to the grating shifts the peak gain to a lower beam energy closer to the beam-wave velocity synchronism. The point of maximum gain for case-B moves to a lower beam energy than case-A, and the peak for case-C moves even lower, while for case-D the gain peak moves further away from the beam-wave velocity synchronism. These effects of beam-grating separation on the gain function are in agreement with the theoretical predictions illustrated in Figs. 12 and 17.

We also note that the experimentally-observed gainversus-energy function when the beam is moved closer

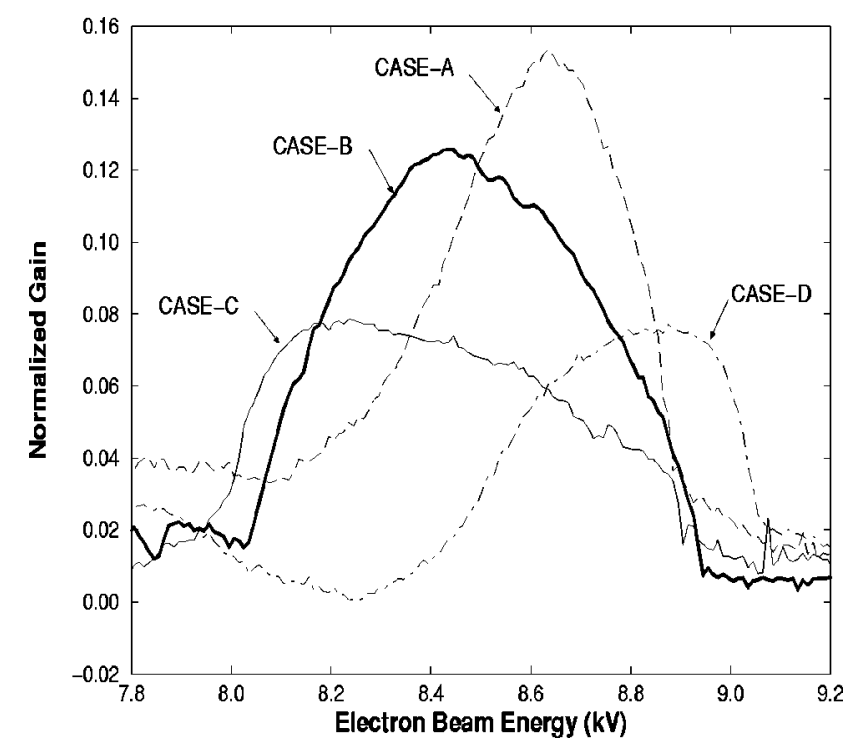

FIG. 19. Experimental gain for several electron beam separations.
(case-B) to the grating is broader than case-A. An explanation for this characteristic is that the strong interaction for the slow-surface-wave is with the core of the electron beam. The core of the electron beam has a lower current-density gradient and, hence, a flatter profile. The uniform-beam-profile theoretical model more closely describes the interaction for case-B. This results in a broader gain shape and a closer agreement with theory. Thus it is experimentally verified that the electron-beam's non-uniform radial density profile is the major influence for the voltage narrowing of the gain function.

\section{SUMMARY}

Experimental measurements of low-beam-energy gain of the rectangular waveguide grating Traveling Wave Tube (TWT) amplifier have been described. The shape of the gain curve and physics of interaction between the microwave fields and the electron beam have been presented for different beam velocity spreads and several beam-grating separations. A normalized single-pass maximum gain of 0.16 was measured at $8.6 \mathrm{kV}$ with a 0.036 velocity spread in the electron beam at the operating frequency of $13.0 \mathrm{GHz}$ and the peak gain matches the theoretical prediction. The presence of a region of enhanced convective growth is discussed and experimentally verified with increased effective interaction length in a multiple-pass system. A nonuniform peaked (Gaussian) radial current density profile was shown to lead to a narrowing of the gain-versus-energy shape. Experimental gain curves for several beam-grating separations were presented to explore the associated changes in the point of peak gain in the gain curve and the narrowing of the gain shape.

\section{ACKNOWLEDGMENTS}

This work was supported by the Office of Naval Research as managed by the Naval Research Laboratories (Grant N000 14-95-1-G022), the Department of Defense Vacuum Electronics Initiative as managed by the Air Force Office of Scientific Research (Grant AFOSR-91-0381), by a National Science Foundation Presidential Young Investigator Award (ECS-9057675), and by the College of Engineering, University of Wisconsin-Madison.

${ }^{1}$ F. S. Rusin and G. D. Bogomolov, Proc. IEEE 57, 720 (1969).

${ }^{2}$ E. M. Marshall, P. M. Phillips, and J. E. Walsh, IEEE Trans. Plasma Sci. 16, 199 (1988).

${ }^{3}$ G. Dohler, D. Gallagher, F. Scafuri, and D. Wortman, Proceedings International Electron Devices Meeting (IEEE) (Institute of Electrical and Electronics Engineers, Piscataway, NJ, 1987), pp. 303-306.

${ }^{4}$ J. Joe, J. Scharer, J. Booske, and B. McVey, Phys. Plasmas 1, 176 (1994).

${ }^{5}$ R. F. Harrington, in Time-harmonic Electromagnetic Fields (McGrawHill, New York, 1961), pp. 152-158.

${ }^{6}$ B. D. McVey, M. A. Basten, J. H. Booske, J. Joe, and J. E. Scharer, IEEE Trans. Microwave Theory Tech. 42, 995 (1994).

${ }^{7}$ M. A. Basten, J. H. Booske, and J. Anderson, IEEE Trans. Plasma Sci. 22, 960 (1994).

${ }^{8}$ J. Joe, L. J. Louis, J. Scharer, J. Booske, and M. Basten, Phys. Plasmas 4, 2707 (1997).

${ }^{9}$ T. C. Marshall, in Free-Electron Lasers (Macmillan, New York, 1985), p. 38.

${ }^{10}$ R. J. Briggs, in Electron-Stream Interaction with Plasmas (M.I.T. Press, Cambridge, 1964), pp. 8-38.

${ }^{11}$ A. R. Curren, K. A. Jenson, and R. F. Roman, Secondary Electron Emis- 
sion Characteristics of Molybdenum-masked, Ion-Textured ofhc Copper (National Aeronautics and Space Administration (NASA), Washington, D.C.), Technical Paper, Cleveland (1990).

${ }^{12}$ G. D. Simms and I. M. Stephenson, Microwave Tubes and Semiconductor Devices (Blackie and Son, London, 1963), pp. 66, 67.
${ }^{13}$ A. W. H. Beck, Space Charge Waves and Slow Electromagnetic Waves (Pergamon Press, New York, 1958), pp. 99-155.

${ }^{14}$ A. Grover and P. Sprangle, IEEE J. Quantum Electron. QE-17, 1196 (1981).

${ }^{15}$ C. W. Roberson and P. Sprangle, Phys. Fluids B 1, 9 (1989). 